doi:10.13108/2020-12-4-114

\title{
LIOUVILLE-TYPE THEOREMS FOR FUNCTIONS OF FINITE ORDER
}

\author{
B.N. KHABIBULLIN
}

\begin{abstract}
A convex, subharmonic or plurisubharmonic function respectively on the real axis, on a finite dimensional real of complex space is called a function of a finite order if it grows not faster than some positive power of the absolute value of the variable as the latter tends to infinity. An entire function on a finite-dimensional complex space is called a function of a finite order if the logarithm of its absolute value is a (pluri-)subharmonic function of a finite order. A measurable set in an $m$-dimensional space is called a set of a zero density with respect to the Lebesgue density if the Lebesgue measure of the part of this set in the ball of a radius $r$ is of order $o\left(r^{m}\right)$ as $r \rightarrow+\infty$. In this paper we show that convex function of a finite order on the real axis and subharmonic functions of a finite order on a finite-dimensional real space bounded from above outside some set of a zero relative Lebesgue measure are bounded from above everywhere. This implies that subharmonic functions of a finite order on the complex plane, entire and subharmonic functions of a finite order, as well as convex and harmonic functions of a finite order bounded outside some set of a zero relative Lebesgue measure are constant.
\end{abstract}

Keywords: entire function, subharmonic function, pluri-subharmonic function, convex function, harmonic function of entire order, Liouville theorem.

Mathematics Subject Classification: 32A15, 30D20, 31C10, 31B05, 31A05, 26B25, 26A51

The base of this work is a classical Liouville theorem for entire functions, that is, for holomorphic on complex plane $\mathbb{C}$ or on $\mathbb{C}^{n}$, where $n \in \mathbb{N}:=\{1,2, \ldots\}$ functions.

Liouville theorem. A bounded entire function is constant.

The same statement holds for bounded from above subharmonic functions on $\mathbb{C}$ [1, Cor. 2.3.4] and as an obvious corollary, for plurisubharmonic functions on $\mathbb{C}^{n}$, convex functions on the real line $\mathbb{R}$ and as an immediate corollary, on $\mathbb{R}^{m}$ with $1<m \in \mathbb{N}$, as well as for harmonic functions on $\mathbb{R}^{m}$ for all $m \in \mathbb{N}[2$, Thm. 1.19].

Recently in work [3, Lm. 4.2], there was given a version of Liouville theorem for entire functions of finite order on $\mathbb{C}$ bounded not everywhere but only outside some small set $E \subset \mathbb{C}$. In [4, Lm. 4.2], its proof was corrected and before its formulation in Theorem 2.1 in [5] it was said that this theorem was established by A.A. Borichev. The proofs in [3] and [4] employ rather advanced facts and arguing from the theory of complex variable and the potential theory on the complex plane.

Theorem B. ([3, Lm. 4.2], [4, Lm. 4.2], [5, Thm. 2.1]) If an entire function of a finite order on $\mathbb{C}$ is bounded outside some set $E \subset \mathbb{C}$ measurable by the planar Lebesgue measure $\lambda$

B.N. Khabibullin, Liouville-TYPe theOREMS FOR FUnCTIONS OF FINITE ORDER.

(c)Khabibullin B.N. 2020.

The research is made in the framework of the development program of Scientific and Educational Mathematical Center of Privolzhsky Federal District, additional agreement no. 075-02-2020-1421/1 to agreement no. 075-02-2020-1421.

Submitted September 1, 2020. 
and this set has a zero planar density in the sense that

$$
\lim _{r \rightarrow+\infty} \frac{\lambda(\{z \in E:|z| \leqslant r\})}{r^{2}}=0,
$$

then this function is constant.

The main result of this work develops and extends Theorem $\mathrm{B}$ on plurisubharmonic and entire functions on $\mathbb{C}^{n}$ for all $n \in \mathbb{N}$, as well as on convex and harmonic functions on $\mathbb{R}^{m}$. At the same time, our proof is simpler in the case of entire functions of a single complex variable and it is based on an approach differing from that employed in the former proofs of Theorem B.

Let a function $M$ with values in an extended real line $\overline{\mathbb{R}}:=\mathbb{R} \cup\{ \pm \infty\}$ is defined on a positive half-line $\mathbb{R}^{+}:=\{x \in \mathbb{R}: x \geqslant 0\}$, in $\mathbb{R}^{m}$ or in $\mathbb{C}^{n}$ identified with $\mathbb{R}^{2 n}$, with the Euclidean norm $|\cdot|$, but, generally speaking, outside some closed ball $\bar{B}(r)$ of a bounded radius $r \in \mathbb{R}^{+}$and centered at the origin. The order of the function $M$ at infinity can be defined as [6, Sect. 2.1]

$$
\operatorname{ord}[M]:=\limsup _{|x| \rightarrow \infty} \frac{\ln \left(1+M^{+}(x)\right)}{\ln |x|} \in \mathbb{R}^{+} \cup\{+\infty\},
$$

where $M^{+}: x \mapsto \max \{0, M(x)\}$ is a positive part of the function $M$. The order of entire function $f$ on $\mathbb{C}^{n}$ is defined as order ord $[\ln |f|]$ in the sense of (2).

Definition. (cf. with (1)) A relative upper Lebesgue density a subset $E \subset \mathbb{R}^{m}$ measurable by the Lebesgue measure $\lambda$ on $\mathbb{R}^{m}$ is the quantity

$$
\overline{\mathrm{L}}_{m}(E):=\limsup _{r \rightarrow+\infty} \frac{\lambda(E \cap B(r))}{r^{m}} \in \mathbb{R}^{+} \cup\{+\infty\} .
$$

If in the right hand side of the above identity the usual limit $\lim _{r \rightarrow+\infty}$ is well-defined, we call it simply relative Lebesgure density $\mathrm{L}_{m}(E) \in \mathbb{R}^{+} \cup\{+\infty\}$ of the set $E$. The definitions are obviously extended to $\mathbb{C}^{n}$ identified with $\mathbb{R}^{2 n}$ and the notations are $\overline{\mathrm{L}}_{2 n}$ and $\mathrm{L}_{2 n}$.

Theorem 1. Let $m \in \mathbb{N}$ and $E \subset \mathbb{R}^{m}$ be a subset of zero relative Lebesgue density $\mathrm{L}_{m}(E)=0$ in $\mathbb{R}^{m}$. If a subharmonic function $v$ of a finite order on $\mathbb{R}^{m}$ is bounded from above on $\mathbb{R}^{m} \backslash E$, then

$$
\sup _{\mathbb{R}^{m}} v=\sup _{\mathbb{R}^{m} \backslash E} v<+\infty .
$$

Let $n \in \mathbb{N}$. A function $\mathbb{C}^{n}$ is called plurisubharmonic if its restriction on each complex straight line is a subharmonic function. In particular, as $n=1$, these notions coincide, while each plurisubharmonic function on $\mathbb{C}^{n}$ is subharmonic on $\mathbb{R}^{2 n}$. By Theorem 1 , the classical Liouville theorem for plurisubharmonic and entire functions implies the following statement.

Theorem 2. Let $n \in \mathbb{N}$ and $E \subset \mathbb{C}^{n}$ be a set of zero relative Lebesgue density in $\mathbb{C}^{n}$ in the sense of the above definition on $\mathbb{R}^{2 n}$ identified with $\mathbb{C}^{n}$, that is, $\mathrm{L}_{2 n}(E)=0$. If a plurisubharmonic or entire function of a finite order on $\mathbb{C}^{n}$ is bounded from above on $\mathbb{C}^{n} \backslash E$, then it is constant.

Subharmonic functions on $\mathbb{R}$ are exactly convex functions. For each $m \in \mathbb{N}$, each convex of harmonic function on $\mathbb{R}^{m}$ is also subharmonic. Thus, by Theorem 1 and classical Liouville theorems for convex or harmonic functions on $\mathbb{R}^{m}$ we obtain immediately the following theorem.

Theorem 3. Let $m \in \mathbb{N}$ and $E \subset \mathbb{R}^{m}$ be a set of zero relative Lebesgue density in $\mathbb{R}^{m}$. If a convex or harmonic function of entire order on $\mathbb{R}^{m}$ is bounded from above on $\mathbb{R}^{m} \backslash E$, then it is constant. 
It remains to prove Theorem 1 and we proceed to this.

For $m \in \mathbb{N}, x \in \mathbb{R}^{m}$ and $r \in \mathbb{R}^{+}$by $\bar{B}(x, r):=\left\{x^{\prime} \in \mathbb{R}^{m}:\left|x^{\prime}-x\right| \leqslant r\right\}$ we denote a closed ball in $\mathbb{R}^{m}$ of radius $r$ centered at $x$, and as above, $\bar{B}(r):=\bar{B}(0, r)$. Similar notation is introduced $\mathbb{C}^{n}$ identified with $\mathbb{R}^{2 n}$. For a $\lambda$-integrable function $v: \bar{B}(x, r) \rightarrow \overline{\mathbb{R}}$ we let

$$
\mathrm{B}_{v}(x, r):=\frac{1}{\lambda(\bar{B}(x, r))} \int_{\bar{B}(x, r)} v \mathrm{~d} \lambda=\frac{1}{b_{m} r^{m}} \int_{\bar{B}(x, r)} v \mathrm{~d} \lambda, \quad \mathrm{B}_{v}(r):=\mathrm{B}_{v}(0, r),
$$

where $b_{m}$ is the volume of the unit ball. These are respectively mean functions of $v$ over closed balls $\bar{B}(x, r)$ and $\bar{B}(r)$. The positivity is understood as $\geqslant 0$, the negativity does as $\leqslant 0$.

Lemma 1. Let $0<R \in \mathbb{R}^{+}$and $v$ be a positive $\lambda$-measurable function on a closed ball $\bar{B}(R) \subset \mathbb{R}^{m}, 0<r<R$. Then

$$
\mathrm{B}_{v}(x, R-r) \leqslant\left(1+\frac{r}{R-r}\right)^{m} \mathrm{~B}_{v}(R) \text { for each point } x \in \bar{B}(r) .
$$

Proof. By definition (5) and owing to the positivity of $v$ on $\bar{B}(R)$ and the inclusions $\bar{B}(x, R-$ $r) \subset \bar{B}(R)$ for all $x \in \bar{B}(r)$ we obtain:

$$
\begin{aligned}
\mathrm{B}_{v}(x, R-r) & \stackrel{\sqrt[5]{ }}{=} \frac{1}{b_{m}(R-r)^{m}} \int_{\bar{B}(x, R-r)} v \mathrm{~d} \lambda \leqslant \frac{1}{b_{m}(R-r)^{m}} \int_{\bar{B}(R)} v \mathrm{~d} \lambda \\
& =\frac{b_{m} R^{m}}{b_{m}(R-r)^{m}} \frac{1}{b_{m} R^{m}} \int_{\bar{B}(R)} v \mathrm{~d} \lambda \stackrel{\sqrt[5]{=}}{\left(1+\frac{r}{R-r}\right)^{m} \mathrm{~B}_{v}(R),}
\end{aligned}
$$

and this completes the proof.

By $\operatorname{sbh}(S)$ we denote the class of all subharmonic (locally convex as $m=1$ ) functions on some open neighbourhoods of the set $S \subset \mathbb{R}^{m}$. The role of means over balls in (5) for subharmonic functions is due to the inequality on mean over ball [1], 2]; this inequality characterizes them completely under the upper semi-continuity and local integrability in the sense of Lebesgue measure $\lambda$. In particular,

$$
v(x) \leqslant \mathrm{B}_{v}(x, r) \quad \text { as } v \in \operatorname{sbh}(\bar{B}(x, r)) .
$$

Lemma 2. Let $0<R \in \mathbb{R}^{+}$and $v$ be a subharmonic function on a closed ball $\bar{B}(R) \subset \mathbb{R}^{m}$, $0<r<R$, and $E \subset \bar{B}(r)$ be a $\lambda$-measurable set. Then

$$
\int_{E} v \mathrm{~d} \lambda \leqslant\left(1+\frac{r}{R-r}\right)^{m} \lambda(E) \mathrm{B}_{v^{+}}(R) .
$$

Proof. By inequality (7) on mean over ball we obtain

$$
v(x) \leqslant \mathrm{B}_{v}(x, R-r) \leqslant \mathrm{B}_{v^{+}}(x, R-r) \text { for each point } x \in \bar{B}(r) .
$$

Integrating this inequality over the set $E$ by the Lebesgue measure $\lambda$ gives the inequality

$$
\int_{E} v \mathrm{~d} \lambda \leqslant \int_{E} \mathrm{~B}_{v^{+}}(x, R-r) \mathrm{d} \lambda(x) .
$$

Hence, by inequality (6) in Lemma 1 applied to the integrand with a positive function $v^{+}$in the latter integral, we obtain

$$
\int_{E} v \mathrm{~d} \lambda \leqslant \int_{E}\left(1+\frac{r}{R-r}\right)^{m} \mathrm{~B}_{v^{+}}(R) \mathrm{d} \lambda(x)=\left(1+\frac{r}{R-r}\right)^{m} \mathrm{~B}_{v^{+}}(R) \lambda(E),
$$

and this proves (8). The proof is complete. 
Lemma 3. Let $0<R \in \mathbb{R}^{+}$, and $v$ be a subharmonic function on a closed ball $\bar{B}(R) \subset \mathbb{R}^{m}$. Then for each number $r \in(0, R)$ and for each $\lambda$-measurable subset $E \subset \bar{B}(r)$ we have the inequality

$$
\mathrm{B}_{v}(r) \leqslant \frac{1}{b_{m} r^{m}} \int_{\bar{B}(r) \backslash E} v \mathrm{~d} \lambda+\frac{1}{b_{m}}\left(1+\frac{r}{R-r}\right)^{m} \frac{\lambda(E)}{r^{m}} \mathrm{~B}_{v^{+}}(R) .
$$

Proof. By definition (5),

$$
\mathrm{B}_{v}(r)=\frac{1}{b_{m} r^{m}} \int_{\bar{B}(r) \backslash E} v \mathrm{~d} \lambda+\frac{1}{b_{m} r^{m}} \int_{E} v \mathrm{~d} \lambda,
$$

and by inequality (8) in Lemma 2 applied to the latter integral, we arrive at (9). The proof is complete.

Proof of Theorem 1. We let

$$
M:=\sup _{\mathbb{R}^{m} \backslash E} v \in \mathbb{R} .
$$

Thanks to the boundedness from above of the function $v$ on $\mathbb{R}^{m} \backslash E$, we can consider a subharmonic function $v-M$ negative on $\mathbb{R}^{m} \backslash E$. We apply Lemma 3 for aribtrary $0<r \in \mathbb{R}^{+}$ with $R=2 r$ and with the set obtained by the intersection $E \cap \overline{B(r) \subset \bar{B}}(r)$ as the set $E$ to a subharmonic function $(v-M)^{+} \geqslant 0$, where the first integral in the right hand side in (9) vanishes. As a result we obtain:

$$
\begin{aligned}
\mathrm{B}_{(v-M)^{+}}(r) & \leqslant \frac{1}{b_{m}}\left(1+\frac{r}{2 r-r}\right)^{m} \frac{\lambda(E \cap \bar{B}(r))}{r^{m}} \mathrm{~B}_{(v-M)^{+}}(2 r) \\
& =\frac{2^{m}}{b_{m}} \frac{\lambda(E \cap \bar{B}(r))}{r^{m}} \mathrm{~B}_{(v-M)^{+}}(2 r) \quad \text { for all } 0<r \in \mathbb{R}^{+} .
\end{aligned}
$$

By condition $\mathrm{L}_{m}(E)=0$ for the function

$$
r \underset{0<r \in \mathbb{R}^{+}}{\longmapsto} \mathrm{B}_{(v-M)^{+}}(r) \in \mathbb{R}^{+}
$$

this yields

$$
\mathrm{B}_{(v-M)^{+}}(r)=o\left(\mathrm{~B}_{(v-M)^{+}}(2 r)\right) \quad \text { as } r \rightarrow+\infty .
$$

Function (11) is of a finite order ord $\left[\mathrm{B}_{(v-M)^{+}}\right] \in \mathbb{R}^{+}$since ord $\left[(v-M)^{+}\right] \in \mathbb{R}^{+}$thanks to the finiteness of the order ord $[v]$. Hence, 12 is possible only in the case $\mathrm{B}_{(v-M)^{+}} \equiv 0$ and, as an implication, $(v-M)^{+} \equiv 0$. Together with (10) this implies (4). The proof is complete.

Remark. The condition of zero Lebesgue density $\mathrm{L}_{m}(E)=0$ in Theorems 1 and 3 , as well as the same condition with $m:=2 n$ in Theorem 2, can be replaced by a formally weaker condition: there exists an unbounded sequence of positive numbers $\left(r_{k}\right)_{k \in \mathbb{N}}$, for which

$$
\limsup _{k \rightarrow \infty} \frac{r_{k+1}}{r_{k}}<+\infty \text { and } \lim _{k \rightarrow \infty} \frac{\lambda\left(E \cap B\left(r_{k}\right)\right)}{r_{k}^{m}}=0,
$$

since the latter implies $\mathrm{L}_{m}(E)=0$.

The author is deeply grateful to A.D. Baranov. Exactly thanks to his very informative plenary report and stimulating online contacts during International scientific conference "Complex analysis and applications" in Kazan in August 24-28, 2020, this short paper arose. 


\section{BIBLIOGRAPHY}

1. Th. Ransford. Potential theory in the complex plane. Cambridge University Press, Cambridge (1995).

2. W.K. Hayman, P.B. Kennedy. Subharmonic functions. Academic Press, London (1976).

3. A. Baranov, Yu. Belov, A. Borichev. Summability properties of Gabor expansions // J. Funct. Anal. 274:9, 2532-2552 (2018).

4. A. Baranov, Y. Belov, A. Borichev. Summability properties of Gabor expansions // Preprint: arXiv:1706.05685v2.

5. A. Aleman, A. Baranov, Y. Belov, H. Hedenmalm. Backward shift and nearly invariant subspaces of Fock-type spaces // Preprint: arXiv: 2007.06107.

6. B.N. Khabibullin, A.V. Shmeleva. Balayage of measures and subharmonic functions to a system of rays. I: The classical case // Alg. Anal. 31:1, 156-210 (2019). [St. Petersbg. Math. J. 31:1, $117-156$ (2020).]

Bulat Nurmievich Khabibullin

Bashkir State University,

Zaki Validi str. 32,

450000, Ufa, Russia

E-mail: khabib-bulat@mail.ru 